\title{
Factors related to renal cortical atrophy development after glucocorticoid therapy in IgG4-related kidney disease: a retrospective multicenter study
}

Ichiro Mizushima ${ }^{1,2}$, Motohisa Yamamoto $^{3}$, Dai Inoue ${ }^{4}$, Shinichi Nishi ${ }^{5}$, Yoshinori Taniguchi ${ }^{6}$, Yoshifumi Ubara ${ }^{7}$, Shoko Matsui ${ }^{8}$, Tetsuhiko Yasuno ${ }^{9}$, Hitoshi Nakashima ${ }^{9}$, Hiroki Takahashi ${ }^{3}$, Kazunori Yamada ${ }^{1}$, Hideki Nomura ${ }^{10}$, Masakazu Yamagishi ${ }^{11}$, Takao Saito ${ }^{9}$ and Mitsuhiro Kawano ${ }^{1 *}$ (1)

\begin{abstract}
Background: In immunoglobulin G4-related kidney disease (IgG4-RKD), focal or diffuse renal cortical atrophy is often observed in the clinical course after glucocorticoid therapy. This study aimed to clarify the factors related to renal atrophy after glucocorticoid therapy in IgG4-RKD.

Methods: We retrospectively evaluated clinical features including laboratory data and computed tomography (CT) findings before and after glucocorticoid therapy in 23 patients diagnosed with IgG4-RKD, all of whom were followed up for more than 24 months.

Results: Seventeen patients were men, and six were women (average age 62.0 years). Average follow-up period was 54.9 months. The average estimated glomerular filtration rate (eGFR) at diagnosis was $81.7 \mathrm{~mL} / \mathrm{min} / 1.73 \mathrm{~m}^{2}$. All patients had had multiple low-density lesions on contrast-enhanced CT before glucocorticoid therapy, and showed disappearance or reduction of these lesions after it. Pre-treatment eGFR and serum lgE level in 11 patients in whom renal cortical atrophy developed 24 months after the start of glucocorticoid therapy were significantly different from those in 12 patients in whom no obvious atrophy was found at that time $\left(68.9 \pm 30.1 \mathrm{vs} 93.5 \pm 14.1 \mathrm{~mL} / \mathrm{min} / 1.73 \mathrm{~m}^{2}\right.$, $P=0.036$, and $587 \pm 254$ vs $284 \pm 263 \mathrm{IU} / \mathrm{mL}, P=0.008$, respectively). Pre-treatment eGFR and serum lgE level were also significant risk factors for renal atrophy development 24 months after the start of therapy with an odds ratio of 0.520 (per $10 \mathrm{~mL} / \mathrm{min} / 1.73 \mathrm{~m}^{2}, 95 \%$ confidence interval (CI) $0.273-0.993, P=0.048$ ) and 1.090 (per $10 \mathrm{IU} / \mathrm{mL}, 95 \%$ Cl: $1.013-1.174, P=0.022$ ), respectively, in age-adjusted, sex-adjusted, serum IgG4 level-adjusted logistic regression analysis. Receiver operating characteristic curve analysis showed that eGFR of less than $71.0 \mathrm{~mL} / \mathrm{min} / 1.73 \mathrm{~m}^{2}$ and serum IgE of more than $436.5 \mathrm{IU} / \mathrm{mL}$ were the most appropriate cutoffs and yielded sensitivity of $63.6 \%$ and specificity of $100 \%$, and sensitivity of $90.9 \%$ and specificity of $75.0 \%$, respectively, in predicting renal atrophy development.
\end{abstract}

Conclusions: This study suggests that pre-treatment renal insufficiency and serum IgE elevation predict renal atrophy development after glucocorticoid therapy in lgG4-RKD.

Keywords: IgG4-related disease, IgG4-related kidney disease, Atrophy, Glucocorticoid

\footnotetext{
* Correspondence: sk33166@gmail.com

'Division of Rheumatology, Department of Cardiovascular and Internal

Medicine, Kanazawa University Graduate School of Medicine, Takara-machi

13-1, Kanazawa, Ishikawa 920-8640, Japan

Full list of author information is available at the end of the article
} 


\section{Background}

Immunoglobulin G4 (IgG4)-related disease (IgG4-RD) is a recently recognized systemic fibro-inflammatory disorder that can affect almost all organs in the body $[1,2]$. It frequently causes various renal lesions, which are collectively referred to as IgG4-related kidney disease (IgG4-RKD) [3]. Many studies have clarified the clinical, radiographic, and histopathological features of this disease [3-6].

Clinical and histopathological responses to glucocorticoid therapy in IgG4-RKD have been characterized by several recent studies [7-10], and the prognosis of this disease is not always favorable. Although glucocorticoid therapy results in a rapid improvement of renal function and radiologic findings within one month after the start of therapy in most cases of IgG4RKD, recovery of renal function is suboptimal in the patients with moderate to severe renal dysfunction before therapy [8]. Moreover, in the clinical course after glucocorticoid therapy, focal or diffuse renal cortical atrophy is observed in a considerable proportion of treated patients. However, the factors related to such renal atrophy have not been well-clarified. This state of affairs prompted us to undertake the present study to clarify the factors related to renal atrophy after glucocorticoid therapy in IgG4-RKD.

\section{Methods}

\section{Patients and materials}

Between 1 January 2007 and July 2015, 27 patients with IgG4-RD were enrolled as candidates for this study from seven collaborating institutions in Japan. Among them, we identified 23 patients with IgG4-RKD with typical renal radiological findings, whose follow-up period was more than 24 months, and who had sufficient follow-up data (Table 1). We diagnosed them as having IgG4-RKD based on their fulfillment of the criteria proposed by the Japanese Society of Nephrology [3] and exclusion of other diseases. All patients had IgG4-related involvement of more than one extra-renal organ.

Renal biopsy was performed in 11 patients, and biopsy of the affected extra-renal organs including salivary and lacrimal glands in the other 10 patients. All of these 21 patients were diagnosed with definite IgG4-RKD with typical histopathological and immunohistochemical findings. The remaining two patients fulfilled the diagnostic criteria of autoimmune pancreatitis (AIP) [11] and IgG4-related dacryoadenitis and sialoadenitis (IgG4-DS) [12], respectively, although they were diagnosed with possible IgG4-RKD. We retrospectively evaluated the clinical features including laboratory data and imaging findings at baseline and during the follow-up period in these 23 patients. The clinical course of all patients was compatible with that of typical IgG4-RD patients, including an initial good response to glucocorticoid. Five
Table 1 Baseline clinical characteristics of 23 patients with IgG4-related kidney disease

\begin{tabular}{|c|c|}
\hline Characteristic & Value ( $\mathrm{n}=23$ patients) \\
\hline Age, years & $62.0 \pm 12.0$ \\
\hline Gender, male (\%) & 73.9 \\
\hline Follow-up period (months) & $54.9 \pm 22.8$ \\
\hline Allergy (\%) & 65.2 \\
\hline Number of extra-renal organs & $3.0 \pm 1.3$ \\
\hline $\operatorname{lgG} 4(\mathrm{mg} / \mathrm{dL})$ & $1069 \pm 533$ \\
\hline $\lg G(\mathrm{mg} / \mathrm{dL})$ & $3021 \pm 1243$ \\
\hline $\operatorname{lgE}(\mathrm{IU} / \mathrm{mL})$ & $429 \pm 296$ \\
\hline Hypocomplementemia (\%) & 34.8 \\
\hline $\mathrm{CRP}(\mathrm{mg} / \mathrm{dL})$ & $0.25 \pm 0.35$ \\
\hline $\mathrm{Cr}(\mathrm{mg} / \mathrm{dL})$ & $1.00 \pm 0.48$ \\
\hline eGFR (mL/min/1.73 m²) & $81.7 \pm 25.8$ \\
\hline Initial dose of PSL (mg/kg/day) & $0.57 \pm 0.17$ \\
\hline Other immunosuppressants (\%) & 21.7 \\
\hline ARB (\%) & 21.7 \\
\hline ACEl (\%) & 0 \\
\hline Relapse (\%) & 34.8 \\
\hline Diabetes mellitus (\%) & 30.4 \\
\hline Hypertension (\%) & 21.7 \\
\hline Ischemic heart disease (\%) & 4.3 \\
\hline Cerebral vascular disease (\%) & 0 \\
\hline Smoking habit (\%) & 52.4 \\
\hline
\end{tabular}

Conversion factor for serum creatinine at diagnosis $(C r) \mathrm{mg} / \mathrm{dL}$ to $\mu \mathrm{mol} / \mathrm{L}$, $\times 88.4$. $A C E$ l angiotensin converting enzyme inhibitor, $A R B$ angiotensin II receptor blocker, CRP serum C-reactive protein at diagnosis, eGFR estimated glomerular filtration rate at diagnosis, $I g G$ serum immunoglobulin $G$ at diagnosis, IgG4 serum immunoglobulin G4 at diagnosis, IgE serum immunoglobulin $\mathrm{E}$ at diagnosis, PSL prednisolone

patients (patients 3, 9, 15, 19, and 20 in Additional file 1: Table S1) had been included in our earlier study [13] and another four patients (patients 13, 17, 22, and 23 in Additional file 1: Table S1) in another earlier study [7].

\section{Imaging evaluation}

All patients underwent whole-body computed tomography $(\mathrm{CT})$ examination at the initial diagnosis, and had evidence of multiple low-density lesions on contrast-enhanced CT. Follow-up CT data were available for all 23 patients, who received glucocorticoid therapy. A single radiologist with extensive experience in IgG4-RD at Kanazawa University Hospital, who was blinded to other clinical data including renal function, reviewed all imaging data.

The sites of renal and extra-renal involvement were noted at the initial diagnostic $\mathrm{CT}$ imaging, and atrophic change development, improvement of individual preexisting renal lesions, and appearance of any new renal lesions were examined at follow-up CT imaging 
including images within 24 months after the start of treatment and at the last review. All subsequent CT scans within 24 months were routinely performed to follow the clinical course and ascertain the efficacy of treatment, while those from 24 months after the start of treatment to the last review were undertaken for various reasons including routine monitoring or specific clinical indications such as suspected relapse.

Atrophic change in renal lesions was defined as a local dimple in the renal cortex or decrease in the total renal volume compared with the initial baseline scan, as judged by the radiologist, who took careful consideration of the age and constitution of each patient. Radiologic improvement of renal lesions was defined as recovery of renal cortical contrast enhancement without atrophic change as mentioned above at the same site as the lowdensity lesion detected at the initial diagnosis.

\section{Definition of clinical improvement and relapse}

Clinical improvement in IgG4-RKD was defined as stabilization or improvement in renal function in terms of serum creatinine level or estimated glomerular filtration rate (eGFR) and radiologic improvement as mentioned above. Improvement in extra-renal lesions was identified according to changes in symptomatic, radiologic, serologic, and/or histologic features [14, 15]. Relapse of IgG4-RKD was identified by each attendant physician on the basis of a rapid rise in serum creatinine after careful exclusion of other renal diseases, and/ or reappearance or worsening of the radiologic findings. Relapse of extra-renal lesions was defined as reappearance or worsening of symptomatic, radiologic, serologic, or histologic features [14, 15]. In IgG4-RD, re-elevation of serological values such as serum IgG or IgG4 without clinical symptoms or abnormal radiologic findings was not considered as relapse.

\section{Statistical analysis}

Statistical analysis was performed using SPSS V.19. Data are presented as means \pm standard deviation. The significance of differences between groups was determined using the Mann-Whitney $U$ test or Wilcoxon signed rank test, while the significance of differences in frequencies was analyzed with Fisher's exact probability test. For assessment of risk factors for development of atrophy 24 months after the start of therapy, unadjusted and age-adjusted, sex-adjusted, serum IgG4 leveladjusted logistic regression analyses were conducted. Receiver operating characteristic (ROC) curve analysis was performed to test the usefulness of certain parameters for the prediction of renal cortical atrophy and to determine the appropriate cutoff value. Significant differences were defined by $P<0.05$.

\section{Results}

Baseline patient profiles

Baseline clinical characteristics of the 23 patients with IgG4-RKD and typical renal radiological findings are listed in Table 1. There were 17 men and 6 women with an average age of $62 \pm 12$ years (range 34-77). One patient (patient 8 in Additional file 1: Table S1) had been treated with prednisolone at a dose of $7 \mathrm{mg} /$ day for IgG4-DS. None of the other 22 patients had been treated with any immunosuppressant drugs, including glucocorticoids, before the diagnosis of IgG4-RKD. All patients had involvement of one or more extra-renal organs (average $3.0 \pm 1.3$ organs, range $1-7$ ). The salivary gland was involved in 20 patients $(87.0 \%)$, the lacrimal gland in 14 patients $(60.9 \%)$, the pancreas, a perivascular lesion, and the lung in 8 patients respectively for each (34.8\%), the retroperitoneum in 3 patients $(13.0 \%)$, the prostate and hepatic-biliary tract in 2 patients respectively for each (8.7\%), and a nerve in 1 patient (4.3\%). The mean follow-up period of the 23 patients after diagnosis was $54.9 \pm 22.8$ months (range 28-93).

At presentation, all patients had elevated serum IgG4 (average $1069 \pm 533 \mathrm{mg} / \mathrm{dL}$, range 263-2160, normal range <105), which was detected using the assay methods of nephelometry, and IgG (average $3021 \pm$ $1243 \mathrm{mg} / \mathrm{dL}$, range 1756-6729, normal range 870$1700)$. There were 16 patients $(69.6 \%)$ with elevated serum IgE (average $429 \pm 296 \mathrm{IU} / \mathrm{mL}$, range $8-1226$, normal range $<250): 8$ patients $(34.8 \%)$ had hypocomplementemia. The average serum creatinine level was $1.00 \pm 0.48 \mathrm{mg} / \mathrm{dL}$ (range $0.40-2.55$ ), and 10 patients (43.5\%) had elevated serum creatinine exceeding $1.0 \mathrm{mg} / \mathrm{dL}$. The average eGFR calculated on the basis of the Chronic Kidney Disease Epidemiology Collaboration (CKD-EPI) equations, which provide the best estimate for individuals with normal or mildly reduced eGFR [16], was $81.7 \pm 25.8 \mathrm{~mL} / \mathrm{min} / 1.73 \mathrm{~m}^{2}$ (range $17.8-116.6$ ), and 3 patients $(13.0 \%)$ had eGFR $<60 \mathrm{~mL} / \mathrm{min} / 1.73 \mathrm{~m}^{2}$. Average serum C-reactive protein (CRP) was $0.25 \pm$ $0.35 \mathrm{mg} / \mathrm{dL}$ (range $0.0-1.2$ ), and only $2(8.7 \%)$ of $23 \mathrm{pa}-$ tients had elevated serum CRP (CRP $>1 \mathrm{mg} / \mathrm{dL}$ ) (Table 1) (see Additional file 1 for more detail on these data).

CT revealed renal parenchymal low-density lesions in all patients (Fig. 1). These lesions were patchily distributed hypo-attenuated lesions in the renal parenchyma, and were single or multiple, and round or wedgeshaped. Of the 23 patients, 3 also had diffuse thickening of the renal pelvic wall characterized by a smooth intraluminal surface. CT also revealed typical extra-renal lesions mainly in the salivary glands, lacrimal glands, pancreas, periaortic/periarterial tissue, and lung.

Diabetes mellitus (DM) was present in seven patients, hypertension (HT) in five, ischemic heart disease in only one; none of the patients had cerebral vascular 


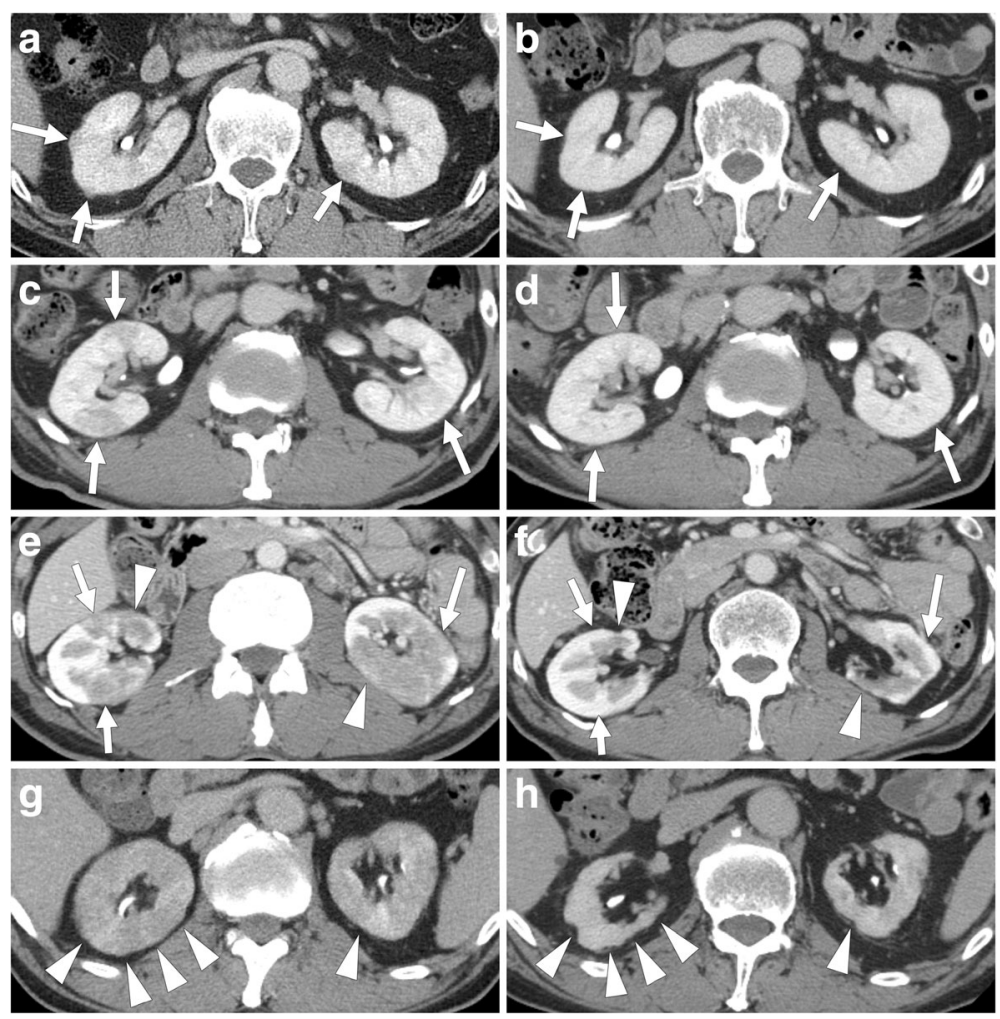

Fig. 1 Low-density lesions after glucocorticoid therapy. The outcome of low-density lesions after glucocorticoid therapy varied between individual patients and even between individual lesions: $\mathbf{a}, \mathbf{c}, \mathbf{e}, \mathbf{g}$ pre-treatment; $\mathbf{b}, \mathbf{d}, \mathbf{f}, \mathbf{h}$ post-treatment. Representative cases are shown: patient 10 (a, $\mathbf{b}$ ); patient 15 (c, d); patient $17(\mathbf{e}, \mathbf{f})$; patient $22(\mathbf{g}, \mathbf{h})$. Patient 10 had small peripheral cortical nodules. Patients 15 and 17 had multiple, round or wedge-shaped lesions. Patient 22 had diffuse patchy involvement. Arrows show recovering lesions, and arrowheads show atrophic lesions

disease. Information on smoking history was available for 21 patients (52.4\%), of whom 11 had a past or current smoking habit.

\section{Treatment}

The indications for treatment and the treatment regimen were decided by the respective attending physician. All 23 patients were treated with prednisolone at an average initial dose of $35.7 \pm 8.3 \mathrm{mg} /$ day (range 20-50) ([0.57 \pm $0.17 \mathrm{mg} / \mathrm{kg} /$ day (range 0.28-0.97)) after the diagnosis. The initial prednisolone dose was continued until 2 to 5 weeks after the start of therapy, and gradually tapered to $2.5-9.0 \mathrm{mg} /$ day in all cases. The average prednisolone dose at the last review was $6.0 \pm 1.7 \mathrm{mg} /$ day. Eighteen patients were treated with glucocorticoid alone during the clinical course, and the remaining five patients with a combination of glucocorticoid and other immunosuppressant drugs such as azathioprine, tacrolimus, or cyclosporine. An angiotensin II receptor blocker (ARB) was administered to five patients for the treatment of hypertension during the clinical course; none of the patients received any angiotensin converting enzyme inhibitors (ACEI).
Clinical and radiological course after glucocorticoid therapy After glucocorticoid therapy, all patients had CT evidence of disappearance or reduction of the low-density lesions during follow up (Fig. 1). Some of the lesions resulted in renal cortical atrophy 24 months after the start of therapy in 11 patients (group A), whereas no obvious atrophy was found at that time in 12 patients (group B).

In five patients treated with glucocorticoid and another immunosuppressant drug during the treatment course, four patients experienced relapse, and one patient had renal atrophy 24 months after the start of therapy. In group A, the mean duration between initiation of treatment and detection of atrophy was $8.9 \pm 5.4$ months. Diffuse thickening of the renal pelvic wall became thinner or normalized after therapy in all of three patients. No development of new renal lesions was observed in any patients under maintenance glucocorticoid therapy during follow up. Three-dimensional (3D) CT was performed in two patients after successful glucocorticoid therapy, and revealed the development of multiple renal cortical scars resembling craters (Fig. 2).

The average eGFR in all 23 patients $(81.7 \pm 25.8 \mathrm{~mL} /$ $\mathrm{min} / 1.73 \mathrm{~m}^{2}$ before therapy) changed to $85.9 \pm 20.7$ at 1 month after the start of therapy, $87.6 \pm 18.0$ at 

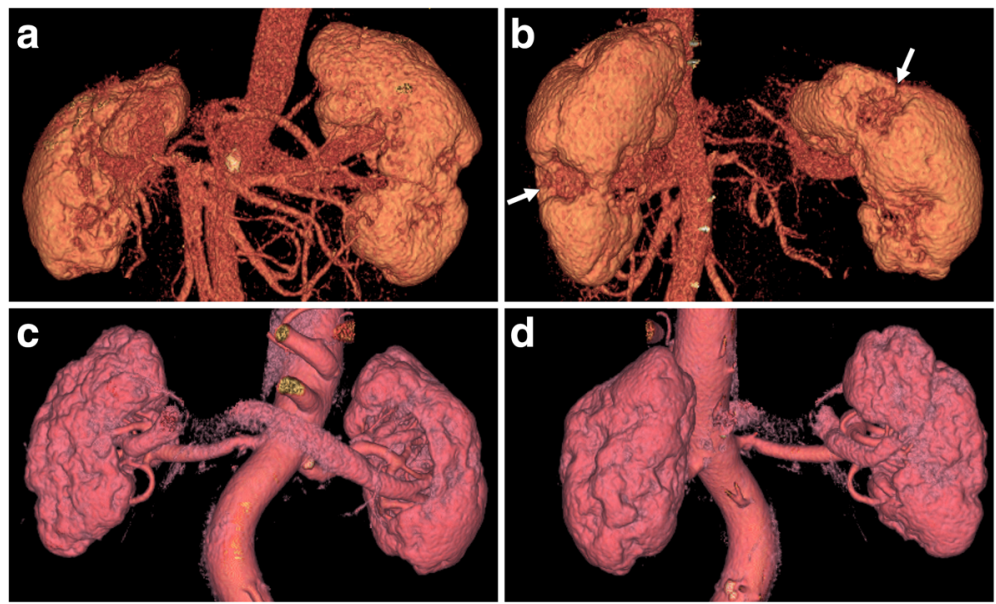

Fig. 2 Three-dimensional computed tomography $(C T)$. Three-dimensional $C T$ revealed the appearance of multiple renal cortical scars resembling craters. Patient 22 (c, d) with worse pre-treatment estimated glomerular filtration rate $\left(35.4 \mathrm{~mL} / \mathrm{min} / 1.73 \mathrm{~m}{ }^{2}\right)$ than patient $17\left(69.3 \mathrm{~mL} / \mathrm{min} / 1.73 \mathrm{~m}{ }^{2}\right)$ $(\mathbf{a}, \mathbf{b})$ had many more cortical scars. Arrows show atrophic lesions

12 months, and $82.0 \pm 17.3$ at the last review. In group $\mathrm{A}$, the average eGFR of $68.9 \pm 30.1 \mathrm{~mL} / \mathrm{min} / 1.73 \mathrm{~m}^{2}$ before therapy changed to $77.5 \pm 22.2$ at 1 month after the start of therapy, $81.8 \pm 22.0$ at 12 months, and $78.2 \pm 21.1$ at the last review. In group B, the average eGFR of $93.5 \pm$ $14.1 \mathrm{~mL} / \mathrm{min} / 1.73 \mathrm{~m}^{2}$ before therapy changed to $93.4 \pm$ 16.6 at 1 month after the start of therapy, $93.3 \pm 12.2$ at 12 months, and $85.5 \pm 13.0$ at the last review.

\section{Factors related to renal cortical atrophy after glucocorticoid therapy}

To identify the factors related to renal atrophy after glucocorticoid therapy, pre-treatment clinical characteristics including co-morbidities, prednisolone dose, other comedication including other immunosuppressant drugs and $\mathrm{ARB}$, and relapse rate were compared between groups A and B (Table 2). Pre-treatment eGFR in group A was significantly lower than that in group B $(68.9 \pm$ 30.1 vs $\left.93.5 \pm 14.1 \mathrm{~mL} / \mathrm{min} / 1.73 \mathrm{~m}^{2}, P=0.036\right)$. Pretreatment serum IgE in group A was significantly higher than that in group B $(587 \pm 254$ vs $284 \pm 263 \mathrm{IU} / \mathrm{mL}, P=$ 0.008). None of the other factors significantly differed between the two groups.

To evaluate the relationship of pre-treatment eGFR and serum IgE level with development of renal atrophy 24 months after the start of therapy, logistic regression analyses were performed for various demographic and clinical variables at baseline. Pre-treatment eGFR and serum IgE level were significant risk factors for development of renal atrophy 24 months after the start of therapy with an odds ratio (OR) of 0.520 (per $10 \mathrm{~mL} / \mathrm{min} /$ $1.73 \mathrm{~m}^{2}, 95 \%$ CI $0.273-0.993, P=0.048$ ) and 1.090 (per $10 \mathrm{IU} / \mathrm{mL}, 95 \%$ CI 1.013-1.174, $P=0.022$ ), respectively, in age-adjusted, sex-adjusted, serum IgG4 level-adjusted logistic regression analysis (Table 3).
To help determine the ability of eGFR and serum IgE to reliably predict the development of renal atrophy after glucocorticoid therapy, and to identify their appropriate cutoffs, we used the ROC curve (Fig. 3). The area under the ROC curve for eGFR was $0.758 \pm 0.111$ (95\% CI $0.539-0.976, P=0.036$ ). We found that eGFR $<71.0 \mathrm{~mL} / \mathrm{min} / 1.73 \mathrm{~m}^{2}$ was the most appropriate cutoff that yielded sensitivity of $63.6 \%$ and specificity of $100 \%$ in predicting the development of renal atrophy (Fig. 3a). The area under the ROC curve for serum IgE was $0.826 \pm 0.091$ (95\% CI $0.647-1.000, P=0.008)$. Serum $\mathrm{IgE}>436.5 \mathrm{IU} / \mathrm{mL}$ was the most appropriate cutoff that yielded sensitivity of $90.9 \%$ and specificity of $75.0 \%$ in predicting development of renal atrophy (Fig. 3b).

\section{Discussion}

We clarified that radiological abnormalities such as focal or diffuse renal cortical atrophy developed despite glucocorticoid therapy during a 2-year clinical course in about $50 \%$ of patients with IgG4-RKD with long-term follow up. In addition, we investigated the factors that determine whether or not radiologic abnormalities leave sequelae, and found that neither the number of involved organs nor serum IgG4 levels, but rather renal function such as eGFR and serum IgE at the time of initiation of glucocorticoid therapy, were related to the development of renal cortical atrophy.

In IgG4-RD, good responsiveness to glucocorticoid is characteristic regardless of which organs are affected $[1,2,4-8,10,15,17]$. Until now, the response to glucocorticoids has been evaluated by means of identifying radiological abnormalities such as tumefactive or hyperplastic lesions, the clinical symptoms caused by them, and functional parameters of the affected organs, including renal function $[8,10]$, salivary secretory function 
Table 2 Comparison of patients with and without renal atrophy 24 months after the start of glucocorticoid therapy

\begin{tabular}{|c|c|c|c|}
\hline & Atrophy $(+)(n=11)$ & Atrophy $(-)(n=12)$ & $P$ value \\
\hline Age (years) & $62.6 \pm 10.7$ & $61.6 \pm 13.5$ & 0.951 \\
\hline Gender (male, \%) & 72.7 & 75.0 & 1.000 \\
\hline Allergy (\%) & 54.5 & 75.0 & 0.400 \\
\hline Number of extra-renal organs & $2.8 \pm 1.0$ & $3.3 \pm 1.6$ & 0.423 \\
\hline $\operatorname{lgG} 4(\mathrm{mg} / \mathrm{dL})$ & $909 \pm 587$ & $1216 \pm 454$ & 0.065 \\
\hline $\operatorname{lgG}(\mathrm{mg} / \mathrm{dL})$ & $3001 \pm 1505$ & $3040 \pm 1015$ & 0.424 \\
\hline $\lg E(I U / m L)$ & $587 \pm 254$ & $284 \pm 263$ & 0.008 \\
\hline $\mathrm{CH} 50(\mathrm{IU} / \mathrm{L})$ & $31.0 \pm 20.5$ & $34.9 \pm 14.8$ & 0.498 \\
\hline CRP $(\mathrm{mg} / \mathrm{dL})$ & $0.31 \pm 0.38$ & $0.19 \pm 0.32$ & 0.251 \\
\hline $\mathrm{Cr}(\mathrm{mg} / \mathrm{dL})$ & $1.23 \pm 0.59$ & $0.80 \pm 0.20$ & 0.052 \\
\hline $\mathrm{eGFR}\left(\mathrm{mL} / \mathrm{min} / 1.73 \mathrm{~m}^{2}\right)$ & $68.9 \pm 30.1$ & $93.5 \pm 14.1$ & 0.036 \\
\hline Initial dose of PSL (mg/kg/day) & $0.52 \pm 0.18$ & $0.62 \pm 0.16$ & 0.124 \\
\hline Other immunosuppressant drugs (\%) & 9.1 & 33.3 & 0.317 \\
\hline ARB (\%) & 27.3 & 16.7 & 0.640 \\
\hline Relapse (\%) & 18.2 & 50.0 & 0.193 \\
\hline Diabetes mellitus (\%) & 36.4 & 25.0 & 0.667 \\
\hline Hypertension (\%) & 27.3 & 16.7 & 0.640 \\
\hline Ischemic heart disease (\%) & 0 & 8.3 & 1.000 \\
\hline Smoking habit (\%) & 50.0 & 54.5 & 1.000 \\
\hline
\end{tabular}

Conversion factor for serum creatinine at diagnosis $(\mathrm{Cr}) \mathrm{mg} / \mathrm{dL}$ to $\mu \mathrm{mol} / \mathrm{L}, \times 88.4$. ARB angiotensin II receptor blocker, $C R P$ serum $\mathrm{C}$-reactive protein at diagnosis, eGFR estimated glomerular filtration rate at diagnosis, IgG serum immunoglobulin $\mathrm{G}$ at diagnosis, $1 g G 4$ serum immunoglobulin $\mathrm{G} 4$ at diagnosis, $I g E$ serum immunoglobulin E at diagnosis, PSL prednisolone

Table 3 Odds ratio for risk of renal atrophy development 24 months after the start of therapy: unadjusted and age-adjusted, sex-adjusted, serum IgG4 level-adjusted logistic regression

\begin{tabular}{|c|c|c|c|c|c|c|}
\hline \multirow[t]{2}{*}{ Variable } & \multicolumn{3}{|c|}{ Unadjusted } & \multicolumn{3}{|c|}{ Age-adjusted, sex-adjusted, serum IgG4 level-adjusted } \\
\hline & $\overline{O R}$ & $95 \% \mathrm{Cl}$ & $P$ value & $\overline{\mathrm{OR}}$ & $95 \% \mathrm{Cl}$ & $P$ value \\
\hline Age (per year) & 1.007 & 0.939 to 1.080 & 0.844 & 1.023 & 0.948 to 1.104 & 0.553 \\
\hline Male sex & 0.889 & 0.138 to 5.723 & 0.901 & 2.045 & 0.228 to 18.311 & 0.522 \\
\hline Allergy & 0.400 & 0.068 to 2.337 & 0.309 & 0.327 & 0.040 to 2.674 & 0.297 \\
\hline Serum lgG4 concentration at baseline (per $10 \mathrm{mg} / \mathrm{dL}$ ) & 0.988 & 0.970 to 1.005 & 0.175 & 0.983 & 0.963 to 1.005 & 0.125 \\
\hline Serum lgG concentration at baseline (per $10 \mathrm{mg} / \mathrm{dL}$ ) & 1.000 & 0.993 to 1.006 & 0.938 & 1.006 & 0.995 to 1.017 & 0.270 \\
\hline Serum IgE concentration at baseline (per $10 \mathrm{IU} / \mathrm{mL}$ ) & 1.056 & 1.004 to 1.111 & 0.035 & 1.090 & 1.013 to 1.174 & 0.022 \\
\hline Serum C3 concentration at baseline (per mg/dL) & 1.001 & 0.977 to 1.025 & 0.957 & 0.995 & 0.967 to 1.024 & 0.718 \\
\hline Serum C 4 concentration at baseline (per mg/dL) & 0.987 & 0.896 to 1.088 & 0.795 & 0.982 & 0.881 to 1.094 & 0.740 \\
\hline Serum CH50 titer at baseline (per IU/L) & 0.987 & 0.940 to 1.036 & 0.585 & 0.980 & 0.928 to 1.034 & 0.452 \\
\hline Serum CRP concentration at baseline (per mg/dL) & 2.776 & 0.208 to 37.079 & 0.440 & 6.149 & 0.273 to 138.380 & 0.253 \\
\hline eGFR at baseline (per $10 \mathrm{~mL} / \mathrm{min} / 1.73 \mathrm{~m}^{2}$ ) & 0.600 & 0.365 to 0.987 & 0.043 & 0.520 & 0.273 to 0.993 & 0.048 \\
\hline Number of organs involved & 0.715 & 0.634 to 1.436 & 0.350 & 0.793 & 0.330 to 1.910 & 0.606 \\
\hline Initial PSL dose (per mg/kg/day) & 0.022 & 0.000 to 6.131 & 0.184 & 0.050 & 0.000 to 54.982 & 0.402 \\
\hline Other immunosuppressant drugs & 0.200 & 0.019 to 2.162 & 0.185 & 0.140 & 0.010 to 2.041 & 0.150 \\
\hline ARB & 1.875 & 0.250 to 14.082 & 0.541 & 1.008 & 0.088 to 11.573 & 0.995 \\
\hline Diabetes mellitus & 1.714 & 0.285 to 10.383 & 0.556 & 1.813 & 0.191 to 17.236 & 0.605 \\
\hline Hypertension & 1.875 & 0.250 to 14.082 & 0.541 & 0.908 & 0.070 to 11.815 & 0.941 \\
\hline Smoking habit & 0.833 & 0.150 to 4.636 & 0.835 & 0.652 & 0.091 to 4.655 & 0.669 \\
\hline
\end{tabular}



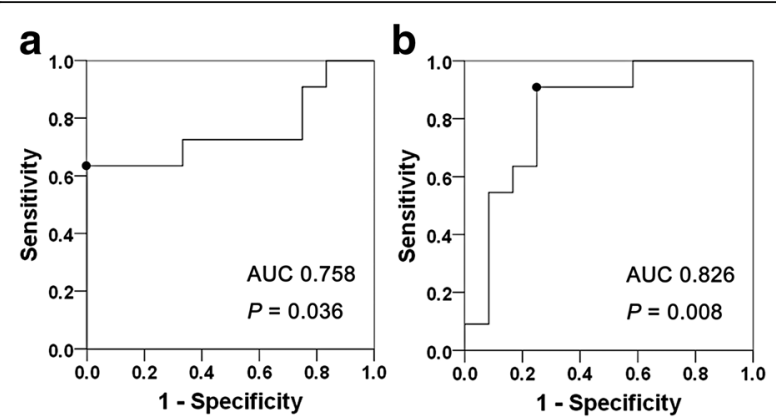

Fig. 3 Receiver operating characteristic curve (ROC) analysis. a ROC curves to identify the appropriate estimated glomerular filtration rate (eGFR) cutoffs for the prediction of renal atrophy development show that eGFR $<71.0 \mathrm{~mL} / \mathrm{min} / 1.73 \mathrm{~m}^{2}$ yields sensitivity of $63.6 \%$ and specificity of $100 \%$ (circle). b ROC curves to identify the appropriate serum lgE level cutoffs for the prediction of renal atrophy development show that serum $\lg \mathrm{E}>436.5 \mathrm{IU} / \mathrm{mL}$ yields sensitivity of $90.9 \%$ and specificity of $75.0 \%$ (circle). AUC area under the curve

[18], and pancreatic exocrine and endocrine function $[19,20]$. Numerous reports reveal that these indicators improve at least initially after the initiation of glucocorticoid therapy. In type 1 AIP, the clinical picture and responsiveness to treatment have been investigated from the earliest period. In this disease, glucocorticoid therapy was reported to promptly achieve reduction of local or diffuse pancreatic swelling, improvement of clinical symptoms, and reduction of serum IgG4 levels [21]. Also in IgG4-RKD, it was shown that radiological abnormalities such as multiple low-density lesions, renal function, and frequently observed hypocomplementemia improved rapidly at the initial stage of glucocorticoid therapy $[7,8]$. Similarly, an initial good response to glucocorticoid is generally recognized in the entire spectrum of IgG4-RD.

However, in contrast to the initial good response to glucocorticoids, recent studies investigating the longterm clinical course of IgG4-RD have revealed that dysfunction of the affected organs can persist. Transformation into chronic pancreatitis associated with pancreatic stones, atrophy, and exocrine and endocrine dysfunction has been reported in the long-term clinical course of type 1 AIP $[19,20]$. A study investigating the long-term clinical course in IgG4-RKD showed that patients with pre-treatment eGFR under $60 \mathrm{~mL} / \mathrm{min} / 1.73 \mathrm{~m}^{2}$ calculated on the basis of the Japanese revised equation for estimating GFR from serum creatinine [22] attained not complete but only partial recovery of renal function one month after the start of glucocorticoid therapy, with residual dysfunction persisting thereafter, and that radiologically, focal or diffuse renal atrophy persisted [8]. Interestingly, renal lesions with complete recovery without any residual atrophy and others with atrophy coexisted radiologically under the circumstances in which global renal function, such as eGFR, improved after glucocorticoid therapy in this study, highlighting the heterogeneity among not only the affected organs but also individual lesions even within the same organ.

The heterogeneity of individual renal lesions in patients with IgG4-RKD was also identified histologically. Raissian et al. analyzed the clinicopathological features of 35 patients with IgG4-related tubulointerstitial nephritis (TIN), and found that the proportion of fibrosis to inflammation varied from specimen to specimen, with some specimens having variability in the extent of fibrosis and inflammation within each tissue sample [6]. In a study comparing light-microscopic findings of IgG4RKD with those of other TIN in detail, Yoshita et al. also reported that all patients with IgG4-RKD had various fibrotic stages within each specimen, confirming the results of the Raissian study [23]. In our earlier study examining the renal histopathology of post-glucocorticoid treatment specimens, we found that in some lesions inflammatory cell infiltrates had disappeared without residual fibrosis, whereas other lesions developed persistent severe fibrosis [7]. This histopathological heterogeneity in the progression of fibrosis may explain why among lesions in which no radiological differences are detected, some recover completely without residual atrophy, whereas others become atrophic.

This study examined the factors related to the development of post-treatment renal cortical atrophy associated with fibrosis, and showed the possibility that pretreatment renal function (eGFR) and serum IgE level could be factors predicting it. Both of these parameters differed significantly between the patients who developed renal atrophy after glucocorticoid therapy and those who did not. Age-adjusted, sex-adjusted, and serum IgG4 level-adjusted logistic regression analysis suggested that they were significantly related to development of renal atrophy. In addition, the usefulness of eGFR and serum IgE to predict the development of renal atrophy was suggested by our ROC curve analysis, in which the area under the ROC curve was 0.758 and 0.826 , showing moderate and high accuracy, respectively. As the patients with eGFR $<71.0 \mathrm{~mL} / \mathrm{min} / 1.73 \mathrm{~m}^{2}$ developed renal atrophy at specificity of $100 \%$, an early initiation of glucocorticoid therapy seems reasonable before eGFR, which is calculated on the basis of CKD-EPI equations, declines to about $70 \mathrm{~mL} / \mathrm{min} / 1.73 \mathrm{~m}^{2}$. Considering the heterogeneity of each lesion in the progression of fibrosis, we can speculate that patients with lower pre-treatment eGFR had already developed more lesions with advanced fibrosis and, hence, such lesions resulted in irreversible atrophic changes despite glucocorticoid therapy. In drug-induced interstitial nephritis, a delay in treatment has been demonstrated to result in the development of fibrosis and worse renal outcomes 
[24]. As the International consensus guidance statement on the management and treatment of IgG4-RD recommends urgent treatment of IgG4-related tubulointerstitial nephritis to prevent irreversible renal failure [25], further study will be necessary to clarify the benefit of early initiation of glucocorticoid therapy in IgG4-RKD.

In addition to pre-treatment eGFR, pre-treatment serum IgE was also identified as a factor significantly related to development of renal atrophy in this study. In contrast to pre-treatment renal function, the impact of serum IgE elevation on renal prognosis has not thus far been documented in IgG4-RKD. Concerning the pathophysiology of IgG4-RD, however, upregulation of T helper (Th) 2 cytokines including interleukin (IL)-4 and IL-13, which promote IgE production, in the affected lesions have been reported [26-29]. Furthermore, it has been indicated that such Th2 cytokines, especially IL-13, play a dominant role in the fibrosis observed in Th2-upregulated conditions $[30,31]$. On the other hand, Wallace et al. reported that baseline serum IgE elevations, in addition to elevations in serum IgG4 and blood eosinophil counts, could be useful predictors of relapses in patients with IgG4-RD treated with rituximab [32], suggesting the significant association between serum IgE and the clinical course or prognosis in this disease. In IgG4-RKD, although the precise etiology of elevated serum IgE remain unclear, it may reflect the upregulation of profibrotic Th2 cytokines that predispose patients to an increased risk of developing renal atrophy.

This study had several limitations. First, the treatment regimen and follow-up protocols were inconsistent among patients because of its retrospective and multiinstitutional nature, complicating the evaluation of the influence of treatment protocol differences on patient outcome. Second, although this study included more patients with long-term, appropriate radiologic follow-up over 2 years than past ones, the number of patients was not sufficient to conclude the identified factors to be definitively significant predictors nor the indicated cutoffs to be optimal in this study. Therefore, larger-scale prospective studies will be needed to confirm our results.

\section{Conclusions}

This study suggests that pre-treatment renal insufficiency and serum IgE elevation can be predictors of renal atrophy after glucocorticoid therapy, and that starting glucocorticoid therapy before eGFR declines to about $70 \mathrm{~mL} /$ $\min / 1.73 \mathrm{~m}^{2}$ may be desirable in IgG4-RKD. Whether earlier initiation of therapy before renal function declines would more consistently prevent the development of renal atrophy remains to be confirmed through a larger-scale prospective study; nevertheless, these observations should be informative for establishing the optimal treatment strategy for IgG4-RKD.

\section{Additional file}

Additional file 1: Table S1. Detailed data of 23 patients with lgG4related kidney disease (PDF 213 kb)

\section{Abbreviations}

3D: three-dimensional; ACEl: angiotensin converting enzyme inhibitor: AIP: autoimmune pancreatitis; ARB: angiotensin II receptor blocker; $\mathrm{Cl}$ : confidence interval; CKD-EPI: Chronic Kidney Disease Epidemiology Collaboration; CRP: C-reactive protein; CT: computed tomography; eGFR: estimated glomerular filtration rate; IgE: immunoglobulin $\mathrm{E}$; IgG: immunoglobulin G; IgG4: immunoglobulin G4; IgG4-DS: immunoglobulin G4 (lgG4)-related dacryoadenitis and sialoadenitis; IgG4-RD: immunoglobulin G4 (lgG4)-related disease; IgG4-RKD: immunoglobulin G4 (lgG4)-related kidney disease; IL: interleukin; OR: odds ratio; ROC: receiver operating characteristic; Th: T helper; TIN: tubulointerstitial nephritis

\section{Acknowledgements}

We thank John Gelblum for his critical reading of the manuscript. The results presented in this paper have not been published previously in whole or part, except in abstract format.

\section{Funding}

This work was supported by Health and Labor Sciences Research Grants for the Study of Intractable Diseases from the Ministry of Health, Labor and Welfare, Japan.

Availability of data and materials

Not applicable.

\section{Authors' contributions}

IM designed the study, acquired data, contributed to analysis and interpretation of data, and drafted the manuscript. MYamamoto acquired data and provided intellectual content of critical importance to the work described. DI contributed to analysis and interpretation of data and provided intellectual content of critical importance to the work described. SN acquired data and provided intellectual content of critical importance to the work described. YT acquired data and provided intellectual content of critical importance to the work described. YU acquired data and provided intellectual content of critical importance to the work described. SM acquired data and provided intellectual content of critical importance to the work described. TY acquired data and provided intellectual content of critical importance to the work described. HNakashima acquired data and provided intellectual content of critical importance to the work described. HT acquired data and provided intellectual content of critical importance to the work described. KY acquired data and provided intellectual content of critical importance to the work described. HNomura contributed to analysis and interpretation of data and provided intellectual content of critical importance to the work described. MYamagishi designed the study, provided intellectual content of critical importance to the work described, and revised the manuscript. TS designed the study and provided intellectual content of critical importance to the work described. MK designed the study, contributed to analysis and interpretation of data, provided intellectual content of critical importance to the work described, and revised the manuscript. All authors read and approved the final manuscript.

\section{Competing interests}

The authors declare that they have no competing interests.

\section{Consent for publication}

Not applicable.

\section{Ethics approval and consent to participate}

This study received institutional ethics board approval from the Medical Ethics Committee of Kanazawa University, institutional review board of Sapporo Medical University Hospital, institutional review committee of Kobe University, Ethical Review Board of Kochi Medical School, institutional review board in Toranomon Hospital, review board of the University of Toyama, and clinical research review committee in Fukuoka University, and informed consent for the use of all data and samples was obtained from each patient. The research was conducted in compliance with the Declaration of Helsinki. 


\section{Author details}

'Division of Rheumatology, Department of Cardiovascular and Internal Medicine, Kanazawa University Graduate School of Medicine, Takara-machi 13-1, Kanazawa, Ishikawa 920-8640, Japan. ${ }^{2}$ Division of Nephrology and Rheumatology, Department of Internal Medicine, Ishikawa Prefectural Central Hospital, Kanazawa, Japan. ${ }^{3}$ The First Department of Internal Medicine, Sapporo Medical University, South 1, West 16, Chuo-ku, Sapporo, Hokkaido 060-8543, Japan. ${ }^{4}$ Department of Radiology, Kanazawa University Graduate School of Medical Science, 13-1 Takara-machi, Kanazawa, Ishikawa 920-8640, Japan. ${ }^{5}$ Division of Nephrology and Kidney Center, Kobe University Graduate School of Medicine, 7-5-1 Kusunoki, Chuo-ku, Kobe, Hyogo 650-0017, Japan. ${ }^{6}$ Department of Endocrinology, Metabolism and Nephrology, Kochi Medical School, Kochi University, Kohasu, Oko-cho, Nankoku, Kochi 783-8505, Japan. ${ }^{7}$ Nephrology Center and Okinaka Memorial Institute for Medical Research, Toranomon Hospital, Toranomon 2-2-2, Minato-ku, Tokyo 105-8470, Japan. ${ }^{8}$ Health Administration Center, University of Toyama, 2630 Sugitani, Toyama-shi, Toyama 930-0194, Japan. ${ }^{9}$ Division of Nephrology and Rheumatology, Department of Internal Medicine, Fukuoka University, 7-45-1, Nanakuma, Jonan-ku, Fukuoka 814-0180, Japan. ${ }^{10}$ Department of General Medicine, Kanazawa University Hospital, 13-1 Takara-machi, Kanazawa, Ishikawa 920-8640, Japan. ${ }^{11}$ Division of Cardiology, Department of Cardiovascular and Internal Medicine, Kanazawa University Graduate School of Medicine, 13-1 Takara-machi, Kanazawa, Ishikawa 920-8640, Japan.

Received: 13 September 2016 Accepted: 8 November 2016 Published online: 25 November 2016

\section{References}

1. Stone JH, Zen Y, Deshpande V. IgG4-related disease. N Engl J Med. 2012;366:539-51.

2. Umehara H, Okazaki K, Masaki Y, Kawano M, Yamamoto M, Saeki T, et al. A novel clinical entity, IgG4-related disease (lgG4RD): general concept and details. Mod Rheumatol. 2012;22:1-14.

3. Kawano M, Saeki T, Nakashima H, Nishi S, Yamaguchi Y, Hisano S, et al. Proposal for diagnostic criteria for lgG4-related kidney disease. Clin Exp Nephrol. 2011;15:615-26.

4. Saeki T, Nishi S, Imai N, Ito T, Yamazaki H, Kawano M, et al. Clinicopathological characteristics of patients with IgG4-related tubulointerstitial nephritis. Kidney Int. 2010;78:1016-23.

5. Nishi S, Imai N, Yoshida K, Ito Y, Saeki T. Clinicopathological findings of immunoglobulin G4-related kidney disease. Clin Exp Nephrol. 2011;15:810-9.

6. Raissian Y, Nasr SH, Larsen CP, Colvin RB, Smyrk TC, Takahashi N, et al. Diagnosis of IgG4-related tubulointerstitial nephritis. J Am Soc Nephrol. 2011;22:1343-52.

7. Mizushima I, Yamada K, Fujii H, Inoue D, Umehara H, Yamagishi M, et al. Clinical and histological changes associated with corticosteroid therapy in IgG4-related tubulointerstitial nephritis. Mod Rheumatol. 2012;22:859-70.

8. Saeki T, Kawano M, Mizushima I, Yamamoto M, Wada Y, Nakashima H, et al. The clinical course of patients with IgG4-related kidney disease. Kidney Int. 2013;84:826-33.

9. Arai H, Hayashi H, Takahashi K, Koide S, Sato W, Hasegawa M, et al. Tubulointerstitial fibrosis in patients with lgG4-related kidney disease: pathological findings on repeat renal biopsy. Rheumatol Int. 2015;35:1093-101.

10. Saeki T, Kawano M, Mizushima I, Yamamoto M, Wada Y, Ubara Y, et al. Recovery of renal function after glucocorticoid therapy for IgG4-related kidney disease with renal dysfunction. Clin Exp Nephrol. 2016;20:87-93.

11. Okazaki K, Kawa S, Kamisawa T, Shimosegawa T, Tanaka M. Japanese consensus guidelines for management of autoimmune pancreatitis: I. Concept and diagnosis of autoimmune pancreatitis. J Gastroenterol. 2010;45:249-65.

12. Masaki Y, Sugai S, Umehara H. IgG4-related diseases including Mikulicz's disease and sclerosing pancreatitis: diagnostic insights. J Rheumatol. 2010;37:1380-5.

13. Mizushima I, Inoue D, Yamamoto M, Yamada K, Saeki T, Ubara Y, et al. Clinical course after corticosteroid therapy in lgG4-related aortitis/periaortitis and periarteritis: a retrospective multicenter study. Arthritis Res Ther. 2014;16:R156.

14. Chari ST, Murray JA. Autoimmune pancreatitis, Part II: the relapse. Gastroenterology. 2008;134:625-8.

15. Kawano M, Yamada K. Treatment of IgG4-related disease. Curr Immunol Rev. 2011;7:246-51.
16. Levey AS, Stevens LA, Schmid CH, Zhang YL, Castro 3rd AF, et al. A new equation to estimate glomerular filtration rate. Ann Intern Med. 2009;150:604-12

17. Sah RP, Chari T. Long term prognosis in IgG4-related systemic disease (ISD). Curr Immunol Rev. 2011;7:5239-45.

18. Shimizu Y, Yamamoto M, Naishiro Y, Sudoh G, Ishigami K, Yajima $H$, et al. Necessity of early intervention for lgG4-related disease-delayed treatment induces fibrosis progression. Rheumatology (Oxford). 2013;52:679-83.

19. Maire F, Le Baleur Y, Rebours V, Vullierme MP, Couvelard A, Voitot H, et al. Outcome of patients with type 1 or 2 autoimmune pancreatitis. Am J Gastroenterol. 2011;106:151-6.

20. Maruyama M, Watanabe T, Kanai K, Oguchi T, Asano J, Ito T, et al. Autoimmune pancreatitis can develop into chronic pancreatitis. Orphanet J Rare Dis. 2014;9:77.

21. Kamisawa T, Shimosegawa T, Okazaki K, Nishino T, Watanabe H, Kanno A, et al. Standard steroid treatment for autoimmune pancreatitis. Gut. 2009;58:1504-7.

22. Matsuo S, Imai E, Horio M, Yasuda Y, Tomita K, Nitta K, et al. Collaborators developing the Japanese equation for estimated GFR. Revised equations for estimated GFR from serum creatinine in Japan. Am J Kidney Dis. 2009:53:982-92.

23. Yoshita K, Kawano M, Mizushima I, Hara S, Ito Y, Imai N, et al. Light-microscopic characteristics of IgG4-related tubulointerstitial nephritis: distinction from nonIgG4-related tubulointerstitial nephritis. Nephrol Dial Transplant. 2012;27:2755-61.

24. González E, Gutiérrez E, Galeano C, Chevia C, de Sequera P, Bernis C, et al. Early steroid treatment improves the recovery of renal function in patients with drug-induced acute interstitial nephritis. Kidney Int. 2008:73:940-6.

25. Khosroshahi A, Wallace ZS, Crowe JL, Akamizu T, Azumi A, Carruthers MN, et al. International Consensus Guidance Statement on the Management and Treatment of IgG4-Related Disease. Arthritis Rheumatol. 2015;67:1688-99.

26. Zen Y, Fujii T, Harada K, Kawano M, Yamada K, Takahira M, et al. Th2 and regulatory immune reactions are increased in immunoglobin G4-related sclerosing pancreatitis and cholangitis. Hepatology. 2007;45:1538-46.

27. Mattoo H, Della-Torre E, Mahajan VS, Stone JH, Pillai S. Circulating Th2 memory cells in IgG4-related disease are restricted to a defined subset of subjects with atopy. Allergy. 2014;69:399-402.

28. Furukawa S, Moriyama M, Tanaka A, Maehara T, Tsuboi H, lizuka M, et al. Preferential M2 macrophages contribute to fibrosis in IgG4-related dacryoadenitis and sialoadenitis, so-called Mikulicz's disease. Clin Immunol. 2015;156:9-18.

29. Takeuchi M, Ohno K, Takata K, Gion Y, Tachibana T, Orita Y, et al. Interleukin 13-positive mast cells are increased in immunoglobulin G4-related sialadenitis. Sci Rep. 2015;5:7696.

30. Wynn TA. Fibrotic disease and the $T(H) 1 / T(H) 2$ paradigm. Nat Rev Immunol. 2004:4:583-94.

31. Fallon PG, Richardson EJ, McKenzie GJ, McKenzie AN. Schistosome infection of transgenic mice defines distinct and contrasting pathogenic roles for IL-4 and IL-13: IL-13 is a profibrotic agent. J Immunol. 2000;164:2585-91.

32. Wallace ZS, Mattoo H, Mahajan VS, Kulikova M, Lu L, Deshpande V, et al. Predictors of disease relapse in IgG4-related disease following rituximab. Rheumatology (Oxford). 2016;55:1000-8.

\section{Submit your next manuscript to BioMed Central and we will help you at every step:}

- We accept pre-submission inquiries

- Our selector tool helps you to find the most relevant journal

- We provide round the clock customer support

- Convenient online submission

- Thorough peer review

- Inclusion in PubMed and all major indexing services

- Maximum visibility for your research

Submit your manuscript at www.biomedcentral.com/submit 